\title{
Hysteresis in cognitive processes
}

\author{
K. Minogue, H. McNamara and D. Flynn
}

\section{INTRODUCTION}

A large amount of the information processing carried out by the brain is carried out outside of and (relatively) independent of the conscious awareness. These nonconscious mechanisms operate at the level of initial acquisition and interpretation of information, and also in the development of complex cognitive frameworks that structure thinking and can be considered key components of personalities. A key feature of these processes is that the individual is not aware of their working, even though they have "learned" something through their action, [1], [2]. The nonconscious processes of interest here are not related to the more wellknown concept of the "unconscious".

While the neuron-level behaviour of the brain during the operation of these nonconscious processes can be studied, the complexity is such that few conclusions can be drawn. It may be more enlightening to study simple models, based on reasonable assumptions and simplifications, that can give insight into the effects and consequences of nonconscious processes. One particular nonconscious process which is of interest is "cognitive bias".

\section{Cognitive Bias}

Cognitive bias is the (possibly inaccurate) interpretation of information using past interpretations and memories, and not through the actual information itself. The action of cognitive bias, for example, leads people to overconfidence, to mistakenly identify spurious correlations and generally to bad judgements in the face of uncertainty. It is an important factor in economic and financial markets, judicial processes and the development of prejudices. "Runaway" bias has been associated with the development of depression. There is some debate as to the extent to which information is processed nonconsiously, but there is convincing arguments that it is a major factor in the acquisition of cognitive bias [3], [4].

This work will discuss the interaction of bias with ambiguous stimuli. Uncertain or ambiguous stimuli are interpreted by the brain using memories of past events, and these will include factors which lead to bias in the interpretation. If an ambiguous stimulus is interpreted (as a result of bias) in support of a bias, the memory of the encounter strengthens the bias. This cycle of stimulus-interpretationencoding is illustrated in figure 1.

Well-developed cognitive bias is difficult to overcome. A substantially unambiguous stimulus (or "shock") in the opposite sense to the bias is required to overcome such a welldeveloped bias. This persistence of effects, and the requirement of a large shock to "wipe-out" a developed state of

K. Minogue, H. McNamara and D. Flynn are with the Department of Applied Mathematics, University College Cork. E-mail: h.mcnamara@ucc.ie

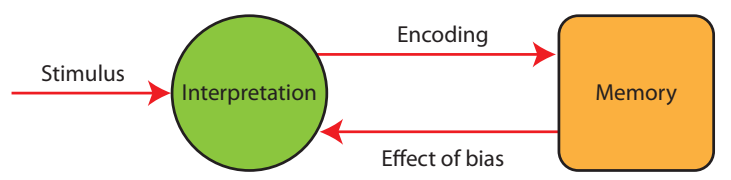

Fig. 1. A schematic of the interpretation of ambiguous stimuli. Cognitive bias, encoded in the memory, effects the interpretation process, and the results of this process may reinforce the bias when encoding in memory takes place.

bias, is suggestive of the mathematical phenomenon of hysteresis. A number of superficial features of memory could also support this view, memory is quite nonlocal (i.e. memories are encoded in a distributed fashion across a large number of neurons), and an emergent result of simpler individual behaviour on the part of neurons (for example it could not be said that a particular neuron "contains" a memory).

This paper will further elaborate the justifications for the use of hysteresis in simple models of cognitive bias. In particular, the classical Preisach model will be used as the "memory" portion of the system illustrated in figure 1 . This will allow for numerical experiments with a simple mathematical system. The qualitative results of this model will be evaluated against the understanding of bias in the psychological literature.

\section{REFERENCES}

[1] A. Reber, "Implicit learning and tacit knowledge," Journal of experimental psychology: general, vol. 118, pp. 219-235.

[2] P. Lewicki, T. Hill, and M. Czyzewska, "Nonconscious acquisition of information," American Psychologist, vol. 47, pp. 796-801, 1992.

[3] D. Kahneman, P. Slovic, and A. Tversky, eds., Judgment under uncertainty: heuristics and biases. Cambridge University Press, 1982.

[4] P. Lewicki, "Nonconscious biasing effects of single instances on subsequent judgments," Journal of Personality and Social Psychology, vol. 48, pp. 563-574, 1985. 